\title{
RADIO DIAGNOSTICS OF GALAXY INTERACTIONS
}

\author{
T.K. MENON \\ Department of Physics and Astronomy \\ University of British Columbia \\ Vancouver, B.C. Canada. V6T 1Z4
}

The Hickson Compact Groups (Hickson 1982) are relatively isolated systems of galaxies with projected separations comparable to the diameters of the galaxies themselves and are an ideal laboratory for the study of the effects of interactions on the various properties of galaxies. Roughly one third of the galaxies in HCG show clear signs of interaction such as tidal distortion, truncation, and peculiar rotation curves. Galaxies that are not now interacting may have suffered past interactions or mergers. My study is based on a VLA survey of galaxies of different morphologies in 65 Compact Groups from Hickson's $(1982,1993)$ catalogue and only groups with minimum of 4 accordant velocities were included in the study. This final sample consisted of 298 galaxies $(80 \mathrm{E}, 92 \mathrm{~S} 0,126 \mathrm{~S})$. The observations were carried out using the VLA in various configurations at wavelengths of $20 \mathrm{~cm}$ and $6 \mathrm{~cm}$ over a period of several years. The angular resolutions ranged from $20^{\prime \prime}$ to $0.3^{\prime \prime}$ and non-detection sensitivities ranged from $1 \mathrm{mJy}$ to $0.3 \mathrm{mJy}$ for different observations. A total of 86 galaxies were detected as radio sources (32ES0, $54 \mathrm{~S})$.

It is found (Menon 1992) that radio-loud ES0 galaxies in the HCG are predominantly the optically brightest galaxies in a group while the detected spirals are more or less uniformly distributed among the top three brightest members of a group. For the vast majority of radio-loud HCG galaxies the radio radiation is mainly from the central regions of those galaxies. I have shown (Menon 1995a) that the median radio luminosity of an isolated spiral (ISPL) sample is about 5 times greater than that of the HCG sample while the median value of the radio to optical luminosity ratio $R$ is about 3 times greater for the ISPL sample. Hence it would appear that statistically the HCG galaxies have lower radio luminosity for a given optical luminosity. For both the ISPL and HCG samples the late type spirals have higher values of $R$ than the earlier types. Quantitatively the median values of $R$ are higher by factors of 2.4 and 5.5 respectively. For the early morphological types the 
difference between the samples is only marginal while for the later types the difference is particularly significant. It should be emphasized that the above comparison is for the total radiation from the two samples. The radio radiation from the HCG sample is in most cases from the nuclear regions only while for the ISPL sample the contribution is mostly from the disks of the galaxies. Hence if we consider the disc emission alone the HCG sample is significantly deficient compared to the ISPL sample. The median value of the ratio $R_{\mathrm{c}}$ of the nuclear radio luminosity to optical luminosity of the HCG spiral sample is about 12 times higher than for the isolated sample. Quantitatively the median values of $R_{\mathrm{c}}$ are about a factor of 3 higher for the earlier spiral types and a factor of 14 higher for the later spiral types. The structure and the spectral indices of the radio emission from these central regions suggest that the central regions of most HCG spirals may be undergoing bursts of star formation.

The results of numerical simulations suggest than during interactions there is both an inflow of gas into the center from the inner regions as well as an outflow of gas from the outer regions. The increase in the central gas density due to the inflows can lead to formation of molecular clouds, increase the frequency of cloud collisions leading to subsequent starburst activity. Such an enhancement of CO emission has been observed in interacting galaxies by Braine \& Combes (1993) and Combes et al. (1994). Recently Leon et al. (1997) have found significant CO emission in HCG spirals similar to that in interacting spirals. The starbursts can then lead to the increase in supernovae resulting in the enhancement of the radio radiation. The greater degree of enhancement of the central component in the case of the later type spirals can be understood on the basis of the fact that the later type spirals have larger amount of gas available in their central regions. For a mean luminosity of $4 \times 10^{20} \mathrm{~W} / \mathrm{Hz}$ at $1.4 \mathrm{GHz}$ the star formation rate turns out to be about $0.1 M_{\odot}$ per year and a supernova rate of 0.02 per year. Even though these rates are not very large for a whole galaxy it may be recalled that in the case of HCG spirals the rate refers only to the nuclear regions. On the other hand the outflow of gas from the outer parts can be expected to lower the magnetic field intensities there due to the strong coupling between the gas and the field. The reported deficiency of neutral hydrogen in a number of HCG spirals by Williams and Rood (1987) may be attributed to a gas outflow of the type implied by the above results. This in turn may be the cause of the decrease in the total nonthermal radio radiation from these interacting systems.

A useful index of the strength of interaction is found to be a combination of the tidal force between the galaxies and the duration of the encounter. The tidal force itself is proportional to $M_{\mathrm{p}} D_{\mathrm{p}}^{-3}$ where $M_{\mathrm{p}}$ is the mass of the perturbing galaxy and $\mathrm{D}_{\mathrm{p}}$ is its perigalactic distance. The duration of the 
encounter can be estimated from the perigalactic distance and the velocity difference between the two galaxies. Parameters of this type have been used by Dahari (1984), Byrd et al. $(1986,1987)$ and Elmegreen et al. (1991) in their study of the effect of interactions among galaxies. The binned values of the sum of the tidal parameters due to all the other members of the group on each galaxy are plotted against $\log R$ for ES0 and S samples in Figure 1. It appears that in the case of ES0 galaxies there is a strong dependence of the radio emission on the total tidal force while in the case of spirals there is no such dependence.

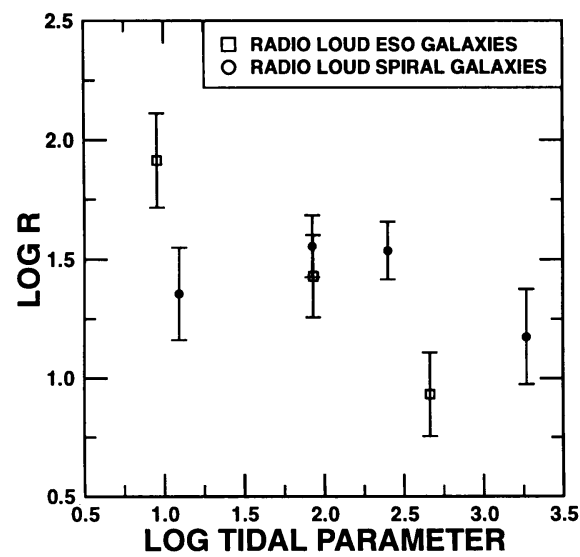

Figure 1. Dependence of $\log R$ on Tidal Parameter

The velocity dispersion of ES0-dominant groups is significantly higher than that of S-dominant groups and this can influence the duration or time scale of the interactions among the galaxies. It is possible that in the case of ES0 galaxies the duration is short enough that recognizable activity manifests itself only when the perturbing galaxy is very close to them producing the close correlation between the separation and activity. On the other hand in the case of $\mathrm{S}$ galaxies the lower velocity dispersion as well as the geometry of disc-halo systems extend both the range and duration over which the tidal forces are effective in initiating the sequence of processes starting with disc instabilities and ending up with inflows into the nucleus and star formation.

Even though the main consequence of interactions among galaxies appears to be the triggering of nuclear activity, numerical simulations suggest that in the case of spiral galaxies specific orbital orientations during the 
encounters can lead to major starburst activity in the spiral arms themselves. The radio maps of the HCG spiral 47a (Menon 1995b) suggest that in the case of this spiral the starburst activity in the spiral arms has been initiated by the tidal action of the companion elliptical galaxy 47b. Since the non-thermal luminosity is most likely produced by relativistic electrons originating in supernova remnants we can calculate the star formation rate to be $\operatorname{SFR}\left(M \geq 5 M_{\odot}\right) \approx 3.7 M_{\odot} \mathrm{yr}^{-1}$. This may be compared to the $\operatorname{SFR}\left(M \geq 5 M_{\odot}\right) \approx 2.2 M_{\odot} \mathrm{yr}^{-1}$ estimated for the whole galaxy M82 by Condon (1992). Since in the present case of HCG47a the volume of the spiral arms producing the radio luminosity is small compared to the galaxy the above rate is indeed very large.

In summary the radio properties of galaxies of different morphological types in high density regions suggest that tidal interaction is most likely the dominant physical process in the initiation of nuclear activity in these systems. In the case of ES0 systems the radio sources generated are generally of the low luminosity FR I type and in the case of spirals nuclear starbursts appear to be the main outcome of the interactions. The absence of any FRII type radio sources in any compact galaxy groups suggests that galaxy interactions alone may not be sufficient to explain the origin of such sources at earlier epochs.

This investigation was supported by a grant from the Natural Sciences and Engineering Research Council of Canada. National Radio Astronomy Observatory is operated by Associated Universities, Inc. under cooperative agreement with the National Science Foundation.

\section{References}

Braine J., \& Combes F., 1993, A \& A, 269, 7

Byrd, G.G., Sundelius, B.\& Valtonen, M.J., 1987. A \& A., 171,16

Byrd, G.G., Valtonen, M.J., Sundelius, B. \& Valtaoja, L.,1986. A \& A., 166,75

Combes F., Prugniel P., Rampazze R., \& Sulentic J., 1994, A \& A, 281, 725

Condon, J.J. 1992, Annual Review of Astronomy and Astrophysics, 30, 575

Dahari, O., 1984. AJ., 89,966

Dahari, O., 1985. ApJS., 57, 643

Elmegreen, D.M., Sundin, M., Elmegreen, B., Sundelius, B. 1991, A \& A, 244, 52

Hickson P., 1982, ApJ., 255, 382

Hickson P., 1993, Ap Lett \& Comm, 29, 1

Menon, T.K., 1991. ApJ., 372,419

Menon T.K., 1992, MNRAS, 255, 41

Menon, T.K., 1995a. MNRAS, 219, 305

Menon, T.K., 1995b. AJ, 110,2605

Williams B.A., \& Rood H.J., 1987, ApJS, 63, 265 